\title{
Prevalencia de Linguatulosis Hepática en Bovinos Beneficiados en una Planta Faenadora de Carnes, Talca, Chile
}

\author{
Prevalence of Bovine Hepatic Linguatuliasis in a Slaughterhouse in Talca, Chile \\ Miguel Carrillo Parraguez ${ }^{1}$, Pamela Morales Muñoz ${ }^{1,4}$, Francisco Carvallo \\ Chaigneau $^{2}$, Cristina Abarca Garrido ${ }^{3}$
}

\section{Resumen}

\begin{abstract}
El objetivo del estudio fue determinar la prevalencia de linguatulosis en hígados de bovinos beneficiados en una planta faenadora de Talca, Chile. Se examinaron 376 hígados y se registró la presencia de lesiones compatibles a linguatulosis, así como la presencia de la ninfa de Linguatula serrata. El 13.8\% de los hígados presentaron lesiones compatibles con linguatulosis y en el $48 \%$ de estos se llegó al diagnóstico etiológico. No se encontraron diferencias significativas por grupo etario $(p=0.187)$. En el $28 \%$ de hígados con diagnóstico etiológico, la linguatulosis fue la única enfermedad parasitaria, en el $60 \%$ estuvo asociada con distomatosis y en un solo caso con un quiste hidatídico. La prevalencia de linguatulosis en hígados de bovinos fue de $6.7 \%$, con base en la presencia de ninfas de L. serrata.
\end{abstract}

Palabras clave: Linguatula serrata, hígado, bovinos, salud pública

\section{Abstract}

The aim of this study was to determine the prevalence of hepatic linguatuliasis of cattle slaughtered in an abattoir in Talca, Chile. Livers $(n=376)$ were examined and compatible lesions and the presence of the nymph of Linguatula serrata were recorded. The results showed that $13.8 \%$ of livers showed lesions compatible with linguatulosis and the etiologic diagnosis was obtained in $48 \%$ of them. No significant differences by age

\footnotetext{
${ }^{1}$ Escuela de Medicina Veterinaria, Facultad de Recursos Naturales y Medicina Veterinaria, Universidad Santo Tomás, Talca, Chile

${ }^{2}$ California Animal Health and Food Safety Laboratory, San Bernardino Branch, USA

${ }^{3}$ Servicio Agrícola y Ganadero Región del Maule, Chile

${ }^{4}$ E-mail:pmoralesm@santotomas.cl
}

Recibido: 1 de junio de 2016

Aceptado para publicación: 25 de octubre de 2016 
group ( $\mathrm{p}=0.187$ ) were found. In $28 \%$ of livers with etiologic diagnosis, linguatuliasis was the only parasitic disease, $60 \%$ was associated with liver fluke and in one case with one hydatid cyst. The prevalence of hepatic linguatuliasis was $6.7 \%$ based on the presence of $L$. serrata nymphs.

Key words: Linguatula serrata, liver, cattle, public health

\section{INTRODUCCIÓN}

La linguatulosis es una enfermedad parasitaria zoonótica, producida por Linguatula serrata (Acha y Szyfres, 2003). Afecta a un gran número de especies, que pueden actuar como hospedadores definitivos (carnívoros domésticos o silvestres) u hospedadores intermediarios (herbívoros, en especial rumiantes) (Caswell y Williams, 2007). El humano puede actuar como hospedador accidental en ambos casos. Se han descrito dos principales formas de linguatulosis, la visceral y la nasofaríngea (Miró, 1999). Ambas formas producen alteraciones de diversa severidad, dependiendo de la especie afectada y de los órganos involucrados (Mehlhorn, 2008a). Si bien la linguatulosis no se cataloga como una enfermedad mortal, se han descrito casos de enfermedad nasofaríngea (Hamid et al., 2012), enfermedad visceral (Lai et al., 2010), enfermedad ocular (Koehsler et al., 2011) e incluso de mortalidad asociada (Ma et al., 2010). Su diseminación se relaciona con las medidas sanitarias del consumo de carne, manejo de subproductos cárnicos y contacto con especies que actúan como hospedadores definitivos, así como con sus secreciones nasales, saliva y heces (Acha y Szyfres, 2003).

En Chile existen escasos estudios sobre la prevalencia de linguatulosis, y todos ellos realizados en décadas pasadas en la región de Los Ríos (Poo, 1971; Garcinuño y González, 1977; Valenzuela et al., 1995). A pesar que esta parasitosis es de importancia en la salud pública y genera pérdidas económicas por el decomiso de órganos en las plantas faenadoras, no se considera en los infor- mes periódicos de decomiso del Servicio Agrícola y Ganadero (SAG). El presente estudio tuvo por objetivo determinar la prevalencia de linguatulosis en hígados de bovinos en una planta faenadora de Talca, mediante el reconocimiento de lesiones compatibles con la parasitosis y la confirmación del parásito por observación de las ninfas. Asimismo, determinar la prevalencia de la enfermedad según el grupo etario de los animales y el tipo de lesiones en el hígado.

\section{Materiales y MéTodos}

\section{Material de Estudio}

Durante los meses de junio y julio de 2015, se examinaron 376 hígados de bovinos beneficiados en una planta faenadora de Talca, Chile. Todos los animales tenían como procedencia la Región del Maule (VII Región), Chile. El número de hígados examinados se determinó aplicando una fórmula para poblaciones finitas (Aguilar-Barojas, 2005), que consideró una población de 1000 animales faenados en dos meses, una confianza del $95 \%$ y una precisión del $4 \%$.

Con base en la cronología dentaria de los animales beneficiados (Luengo et al., 1990), se conformaron cinco grupos etarios (Cuadro 1).

\section{Inspección Hepática}

Los hígados fueron inspeccionados, según el método usado por Valenzuela et al. (1995), comenzando por una inspección visual de toda la superficie hepática y la reali- 
zación de un corte longitudinal en la cara visceral del órgano, comprometiendo hilio y grandes vasos; luego, seis cortes longitudinales paralelos al primero, separados por $2 \mathrm{~cm}$ de distancia entre sí.

El registro del grupo etario y de las lesiones encontradas se efectuó mediante una ficha de muestreo y fotografías. Las lesiones consideradas como compatibles a linguatulosis fueron pequeños nódulos blanco grisáceos de 2 a $4 \mathrm{~mm}$ de diámetro, de márgenes bien delimitadas, de distribución focal o multifocal, y planos o levemente elevados (Garcinuño y González, 1977; Valenzuela et al., 1995). Asimismo, los hallazgos considerados como diagnóstico etiológico fueron las ninfas de L. serrata. El reconocimiento de ninfas se realizó con base en sus estructuras características, como la presencia de espículas en su superficie y cuatro ganchos equidistantes al orificio bucal en el extremo anterior (Mehlhorn, 2008b). Las porciones de hígado compatibles a linguatulosis se recolectaron y preservaron en frascos con alcohol etílico al $70 \%$ para su examinación microscópica.

\section{Análisis Estadístico}

Los datos fueron tabulados en planillas Microsoft Excel y organizados mediante estadística descriptiva. Para determinar diferencias de prevalencias entre grupos etarios se aplicó la prueba no paramétrica de Chi cuadrado de Pearson, usando el programa IMB SPSS ${ }^{\circledR}$ v. 23 para Windows (SPSS Inc, Chicago IL, USA).

\section{Resultados y Discusión}

\section{Lesiones Compatibles a Linguatulosis Hepática}

En el 13.8\% (52/376) de hígados se observaron lesiones compatibles a linguatulosis (Figura 1). Estos resultados difieren de lo descrito por Poo (1971) y Garcinuño y González
(1977) en la región de Los Ríos, quienes mencionan $8.0 \%(\mathrm{n}=483)$ y $5.9 \%(\mathrm{n}=1000)$ de hígados con lesiones compatibles a linguatulosis. La variabilidad entre estos resultados podría explicarse por las diferencias metodológicas utilizadas y el tamaño de la población muestreada. También es importante tomar en consideración las diferencias contextuales cronológicas y presencia de diseminadores silvestres que pudiesen afectar la supervivencia de los estados infectantes del parásito.

En la zona austral de Chile (región de Magallanes), se ha determinado esta parasitosis en hospedadores definitivos de vida silvestre, encontrando una prevalencia de $15.7 \%$ (Alarcón, 2005), pero no se dispone de estudios de prevalencia de esta parasitosis en hospedadores definitivos domésticos en el país. El reconocimiento de lesiones compatibles es lo más accesible en términos prácticos para sospechar de una infestación por L. serrata. Además, esta es la forma en que el médico veterinario diagnostica y dictamina el decomiso. No obstante, el procedimiento se dificulta ante la escasa información científica referente a esta patología y a que en Chile no se le considera como enfermedad de notificación, lo que podría afectar la sospecha de la enfermedad en la inspección hepática de rutina (Norma Chilena 62 de Inspección de Carnes, Ministerio de Salud, 2002).

\section{Distribución y Ubicación de las Lesio- nes}

La caracterización y registro de patrones descriptivos son elementos necesarios para reconocer e interpretar las lesiones observadas (Stromberg, 2005). En este estudio, se observaron 75 focos de lesión en los 52 hígados con lesiones compatibles con linguatulosis. En cuanto a la distribución, 47 órganos $(90.4 \%)$ presentaron uno o dos focos, mientras que 5 (el 9.6\% restante) presentaron 3 o más focos. Estas lesiones, por su pequeño tamaño, pueden pasar inadvertidas durante una inspección de rutina. 
El 81.3\% (61/75) de los focos de lesión se ubicaron inmersos en la cápsula hepática (subcapsulares), mientras que 18.7\% (14/75) se encontraron en el parénquima. Estos resultados son acordes con el estudio de Valenzuela et al. (1995), quienes reportaron el $80 \%$ de lesiones subcapsulares. No obstante, Garcinuño y González (1977) observaron todos los focos en ubicación subcapsular. Esto pudo ser posible ante un error metodológico, ya que los focos subcapsulares son más notorios debido al contraste que generan las lesiones blanco grisáceas en esa ubicación. Las lesiones profundas en el parénquima se pueden confundir y perder en el conjunto de cortes transversales de pequeños canalículos biliares, especialmente cuando se encuentran fibróticos y con tonalidades heterogéneas, condición que se observó en la mayoría de los hígados afectados, debido, en parte, a la alta frecuencia de distomatosis.

\section{Reconocimiento de Ninfas}

En el 48\% (24/52) de los hígados con lesiones compatibles con linguatulosis se encontraron ninfas de L. serrata (Figura 2), lo que permitió llegar al diagnóstico etiológico de la enfermedad. No obstante, la diferencia entre el resultado de prevalencia de linguatulosis basada en lesiones compatibles y la prevalencia basada en la cantidad de lesiones definitivas (presencia de ninfas) fue grande. Una explicación para esto es que algunas lesiones hayan sido antiguas, habiéndose destruido las estructuras ninfales (Yakhchali y Tehrani, 2011), aunque también es posible reconocer que hayan sido producidas por otros agentes.

La presencia de pequeños abscesos en hígados de bovinos como respuesta del tejido hepático a la invasión bacteriana, así como pequeños granulomas formados por estados juveniles de Fasciola hepatica, que en su migración quedan atrapados en el parénquima (Stalker y Hayes, 2007; Cullen y Brown, 2012) son bastante comunes. Hasta el presente, el único estudio en Chile donde se examinó la totalidad de lesiones compatibles con linguatulosis en búsqueda de ninfas fue realizado por Valenzuela et al. (1995) en la región de Los Ríos, donde determinaron el $16.6 \% \quad(n=1011)$ de prevalencia de linguatulosis.

\section{Prevalencia según Grupo Etario}

No se encontraron diferencias significativas para la frecuencia de presentación de linguatulosis entre grupos etarios $(\mathrm{p}=0.187$; Cuadro 1). Esto es acorde con lo expuesto en varios trabajos realizados en Turquía, Irán e India, donde se examinaron tanto hígados como linfonodos mesentéricos de ganado bovino, búfalos, ovejas y camellos (Ravindran et al., 2008; Aydenizoz et al., 2012; Nourollahi Fard et al., 2011, 2012; Alborzi et al., 2013; Dehkordi et al., 2014; Aldemir et al., 2014). Es posible que, independientemente de la edad, los animales estarían expuestos a los huevos infectantes de L. serrata presentes en el agua de bebida, pasturas y suelos a los que acceden.

Por otra parte, existen trabajos que determinan una asociación significativa entre la edad y la presentación de linguatulosis. Nourollahi Fard et al. (2010) y Rezai et al. (2011) reportan una mayor prevalencia en

Cuadro 1. Hígados examinados y positivos a linguatulosis (presencia de ninfas) por grupo etario

\begin{tabular}{ccc}
\hline Grupo & \multicolumn{2}{c}{ Hígados } \\
\cline { 2 - 3 } $\begin{array}{c}\text { etario } \\
\text { (meses de } \\
\text { edad) }\end{array}$ & $\begin{array}{c}\text { Examinados } \\
(\mathrm{n})\end{array}$ & $\begin{array}{c}\text { Positivos } \\
(\mathrm{n})\end{array}$ \\
\hline$<9$ & 16 & 1 \\
10 a 30 & 215 & 18 \\
31 a 52 & 84 & 1 \\
53 a 96 & 54 & 5 \\
$>96$ & 7 & 0 \\
\hline Total & 376 & 25 \\
\hline
\end{tabular}




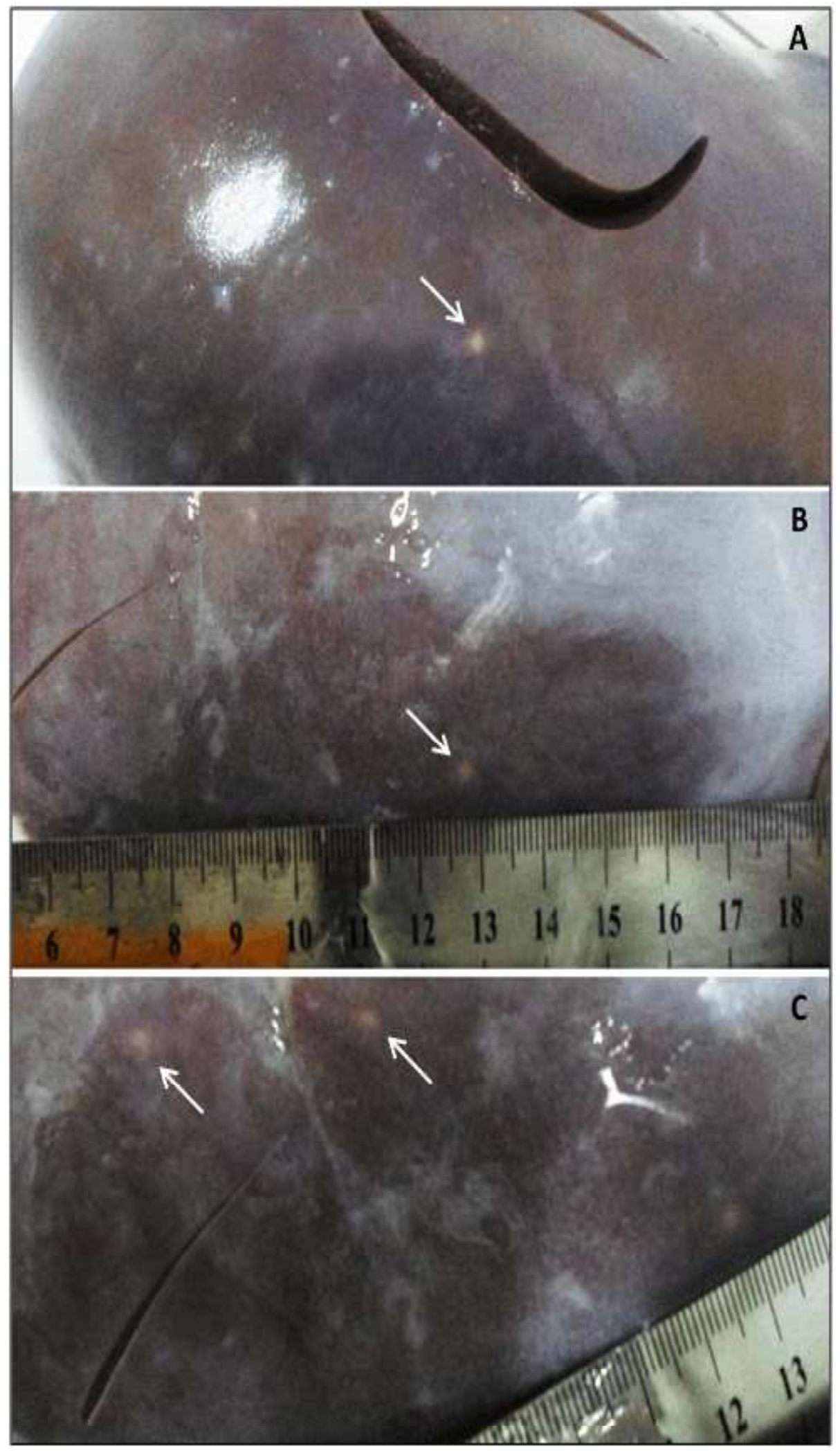

Figura 1. A. Lesión compatible a linguatulosis (flecha) en hígado de un bovino faenado en una planta faenadora de Talca, Chile; B. Lesión compatible a linguatulosis (flecha), donde se aprecia su tamaño; C. Dos focos de lesión compatible a linguatulosis (flechas), próximos entre sí 

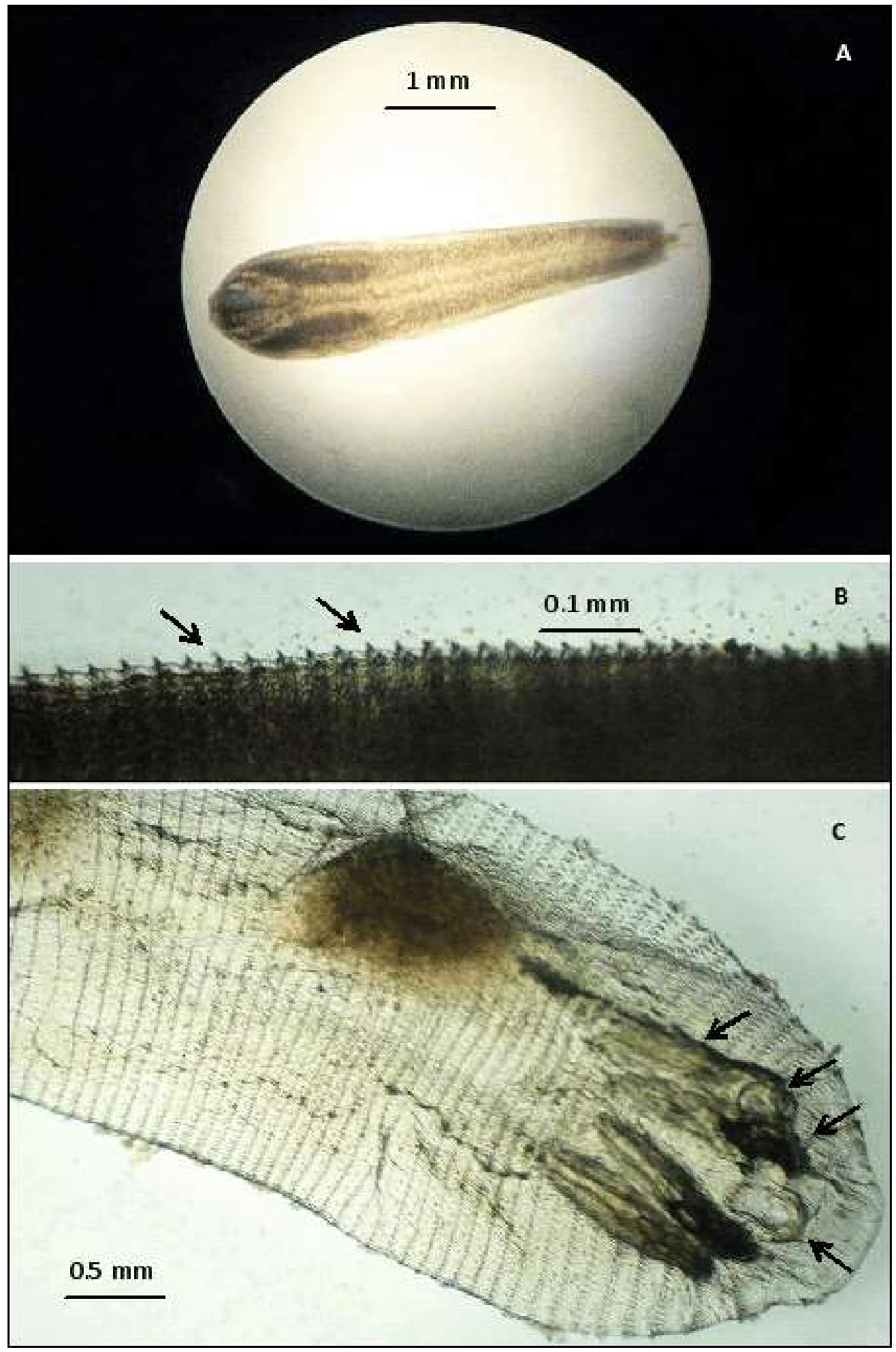

Figura 2. A. Ninfa de L. serrata extraída de una lesión hepática de un hígado inspeccionado en una planta faenadora de Talca, Chile. 10X; B. Parte del cuerpo de ninfa de L. serrata. 100X. Espinas (flechas) en disposición serrada al costado del parásito; C. Extremo anterior de ninfa de L. serrata conservada en alcohol etílico al 70\%. 100X. Nótese (flechas) bulbos y ganchos peribucales y (flecha) abertura bucal 
animales mayores de 36 meses y mayores de 48 meses, respectivamente. Los autores atribuyen este fenómeno a que la ninfa demora de 5 a 7 meses en desarrollarse al estado de lesión infectiva, lo que podría contribuir a la baja prevalencia en las categorías etarias más jóvenes.

\section{Relación con Otros Parásitos}

Se evaluó la presencia de otras lesiones parasitarias, como una forma de contextualizar la presentación de linguatulosis como única enfermedad hepática y en concomitancia con otras enfermedades parasitarias. De los 24 hígados con presencia de ninfas de $L$. serrata, 15 estuvieron asociados con la presencia de $F$. hepatica y un hígado con la presencia de quiste hidatídico.

Asimismo, el 70.7\% (266/376) de los hígados presentaron distomatosis. Este resultado era esperado, ya que la distomatosis se describe como la mayor causa de decomiso hepático en Chile (SAG, 2015). Por otro lado, solo $11(2.9 \%)$ hígados presentaron quistes hidatídicos, frecuencia bastante baja pues se describe a la hidatidosis como la segunda causa de decomiso hepático a nivel nacional (SAG, 2015).

\section{ConClusiones}

- La prevalencia de linguatulosis en hígados de bovinos fue de $13.8 \%$, basándose en el reconocimiento de lesiones compatibles, y de $6.7 \%$, basándose en la presencia de ninfas de Linguatula serrata.

- La mayoría de las lesiones compatibles a linguatulosis tuvieron una ubicación subcapsular y se presentaron en uno o dos focos por hígado.

- La inspección de rutina y el reconocimiento de las lesiones macroscópicas de linguatulosis constituyen una aproximación diagnóstica para su detección; sin embargo, no constituyen un diagnóstico etiológico de la enfermedad.
- La linguatulosis es una enfermedad parasitaria, zoonótica y detectable macroscópicamente a nivel de plantas faenadoras; características importantes para ser consideradas por el Servicio de Salud y registradas en el Informe Anual de Decomisos que genera el Servicio Agrícola y Ganadero en Chile.

\section{Literatura Citada}

1. Acha P, Szyfres B. 2003. Pentastomiases. In: Zoonoses and communicable diseases common to man and animals. Vol III. $3^{\text {rd }}$ ed. Washington DC, USA. p 345-350.

2. Aguilar-Barojas S. 2005. Fórmulas para el cálculo de la muestra en investigaciones de salud. Salud en Tabasco 11(1-2): 333-338.

3. Alarcón U. 2005. Estudio taxonómico de la fauna parasitaria del tracto gastrointestinal de zorro gris (Pseudalopex griseus, Gray 1837), en la XII Región de Magallanes y Antártica Chilena. Tesis de Médico Veterinario. Valdivia, Chile: Universidad Austral de Chile. $45 \mathrm{p}$.

4. Alborzi A, Haddad Molayan P, Akbari M. 2013. Prevalence of Linguatula serrata nymphs in mesenteric lymph nodes of cattle and buffaloes slaughtered in Ahvaz abattoir, Iran. Iran J Parasitol 8: 327-332.

5. Aldemir O, Aydenizoz M, Atesoglu O. 2014. Parasitological and pathological investigations on Linguatula serrata nymphs in mesenteric lymph nodes in sheep in Konya region in Turkey. TURJAF 2: 224-227.

6. Aydenizoz M, Oruc E, Gazyagci A. 2012. Prevalence and pathology of Linguatula serrata infestation in mesenteric lymph nodes of sheep in Kirikkale, Turkey. Isr J Vet Med 67: 102-105.

7. Cullen J, Brown D. 2012. Hepatobiliary system and exocrine pancreas. En: Zachary JF, McGavin MD (eds). 
Pathologic basis of veterinary disease. $5^{\text {th }}$ ed. St Louis MO, USA: Elsevier Saunders. p 431-433.

8. Dehkordi Z, Pajohi-Alamoti M, Azami S, Bahonar A. 2014. Prevalence of Linguatula serrata in lymph nodes of small ruminants: case from Iran. Comp Clin Path 23: 785-788. doi: 10.1007/ s00580-013-1689-5

9. Garcinuño L, González H. 1977. Linguatulosis hepatica en bovinos de Valdivia. Arch Med Vet 9: 62-65.

10. Hamid T, Hossein Y D, Mehran B B, Masood FS, Hamid E. 2012. A case report of Linguatula serrata infestation from rural area of Isfahan City, Iran. Adv Biomed Res 1: 42. doi: 10.4103/22779175.100142

11. Koehsler M, Walochnik J, Georgopoulos $M$, Pruente $C$, Boeckeler W, Auer H, BarisaniAsenbauer T. 2011. Linguatula serrata tongue worm in human eye, Austria. Emerg Infect Dis 17: 870-872. doi: 10.3201/eid1705.100790

12. Lai C, Wang $X$, Lin L, Gao C, Zhang H, Zhang Y, Zhou YB. 2010. Imaging features of pediatric pentastomiasis infection: a case report. Korean J Radiol 11: 480-484. doi: 10.3348/kjr.2010.11.4 .480

13. Luengo J, Aros C, Gómez L. 1990. Determinación de la edad del bovino según las características morfológicas de los dientes incisivos. Contribución a la aplicación de la norma chilena 1423 Of. 84. Terminología y clasificación. Av Cs Vet 5(1). doi:10.5354/0719-5273.1990.-10398

14. Ma K, Qiu M, Rong Y. 2002. Pathological differentiation of suspected cases of pentastomiasis in China. Trop Med Int Health 7: 166-177.

15. Mehlhorn H. 2008a. Linguatulidae. En: Mehlhorn H (ed). Encyclopedia of parasitology. $3^{\text {rd }}$ ed. Nueva York: Springer-Verlag. p 720-721.

16. Mehlhorn H. 2008b. Pentastomida. In: Mehlhorn H (ed). Encyclopedia of parasitology. $3^{\text {rd }}$ ed. Nueva York: Springer-Verlag. p 1114-1124.
17. Ministerio de Salud. 2002. Norma general técnica sobre inspección médico veterinaria de las reses de abasto y de sus carnes y criterios para la calificación de aptitud para el consumo humano. Santiago, Chile. 64 p. [Internet]. Disponible en: http://www.sag.cl/sites/ default/files/norma_62_refundido_aptitud_consumo_-humano.pdf

18. Miró G. 1999. Parasitosis respiratorias y cardiopulmonares: linguatulosis. pneumoniosis. En: Cordero del Campillo M, Rojo F (eds). Parasitología veterinaria. Madrid: McGraw-Hill. p 699-700.

19. Nourollahi Fard S, Kheirandish R, Asl E, Fathi S. 2011. Mesenteric and mediastinal lymph node infection with Linguatula serrata nymphs in sheep slaughtered in Kerman slaughterhouse, southeast Iran. Trop Anim Heath Prod 43: 1-3. doi: 10.1007/s11250-010-9670-7

20. Nourollahi Fard $S$, Kheirandish $R$, Norouzi E, Fathi S. 2010. The prevalence of Linguatula serrata nymphs in mesenteric lymph nodes in cattle. Am J Anim Vet Sci 5: 155-158.

21. Nourollahi Fard S, Ghalekhani N, Kheirandish R, Fathi S, Asl E. 2012. The prevalence of Linguatula serrata nymphs in camels slaughtered in Mashhad slaughterhouse, Northeast, Iran. Asian Pac J Trop Biomed 2: 885888. doi: 10.1016/S2221-1691(12)60247-0

22. Poo L. 1971. Estudio anatomopatológico de los hígados de bovino en el matadero Socoagro de Valdivia. Memoria de título. Valdivia, Chile: Universidad Austral de Chile. 30 p.

23. Ravindran R, Lakshmanan B, Ravishankar C, Subramanian $H$. 2008. Prevalence of Linguatula serrata in domestic ruminants in South India. Southeast Asian J Trop Med Public Health 39: 809-812.

24. Rezaei F, Tavassoli A, Mahmoudian A. 2011. Prevalence of Linguatula serrata infection among dogs (definitive host) and domestic ruminants (intermediate host) in the North West of Iran. Vet Med-Czech 56: 561-567. 
25. [SAG] Servicio Agrícola y Ganadero. 2015. Informe de beneficio y hallazgos patológicos en mataderos nacionales 2014. [Internet]. Disponible en: http:// www.sag.cl/sites/default/files/ informe_decomisos_mataderos_2014.pdf

26. Stalker M, Hayes M. 2007. The liver and biliary system. En: Maxie MG (ed). Jubb, Kennedy, and Palmer's pathology of domestic animals. $5^{\text {th }}$ ed. St Louis MO, USA: Elsevier Saunders. p 298-357.

27. Stromberg P. 2005. Fundamental principles of gross anatomic pathology How to interpret what you see. Ohio
State University. [Internet]. Disponible en: http://www.cldavis.org/cgi-bin/ download.cgi?pid=143.html

28. Valenzuela G, Bascuñan M, Bayer L, Ernst S. 1995. Infecciones por Linguatula serrata (Fröhlich 1789) en hígados de bovinos. Arch Med Vet 27(1): 29-34.

29. Yakhchali M, Tehrani A. 2011. Pathological changes in mesenteric lymph nodes infected with $L$. serrata nymphs in Iranian sheeps. Revue Méd Vét 162: 396-399. 